\title{
Codeine misuse among service users on a methadone treatment programme
}

\author{
D. P. Cohen · E. Unoh · H. Barry · J. J. O'Connor
}

Received: 2 September 2009/Accepted: 26 October 2009/Published online: 21 November 2009

(C) Royal Academy of Medicine in Ireland 2009

Dear Editor,

In recent times, there has been an increased interest in levels of codeine misuse and dependence in the general population that has received widespread media attention [1]. This study aimed to determine the current and past prevalence rates of codeine misuse in service users attending an outpatient methadone stabilisation and detoxification programme from a geographically defined catchment in Dublin. Eighty-nine service users agreed to take part in the study. Information was obtained on demographics, as well as past and current codeine misuse using the Maudsley Addiction Profile [2]. Service user reports of current misuse were confirmed by regular urinalysis.

Thirty-one per cent of participants admitted a past history of codeine misuse. Six per cent $(n=5)$ of participants had a recent history (within the last month) of codeine misuse, with all but one of this group also having a past history of codeine misuse. Those with any history of codeine misuse were more likely to be older (38 vs. 32.9 years, $P<0.05$ ), male ( 82 vs. $69 \%, P=0.09$ ), and have a longer drug dependence history (18.3 vs. 14.9 years, $P<0.05)$. The average duration of misuse was 2.4 years with the most commonly misused substances including codeine phosphate linctus and DF118. The route of administration in all cases was reported as oral.

This study shows that recent codeine misuse in a population attending for methadone stabilisation and detoxification is low. The number of service users reporting a past history is much higher. Further research is required to determine whether the small number is due to regular monitoring of urine samples or due to the substitution of methadone for codeine. However, the profile of those with a past history of codeine misuse (older, longer history of misuse) suggests that contemporaneous trends, i.e. previous availability of codeine or access to stabilisation programmes may explain the discrepancy.

\section{References}

1. Nolan L (2008) Codeine addicts seeking rehab, Sunday Independent, 18 May 2008

2. Marsden J, Gossop M, Stewart D, Best D, Farrell M, Lehmann P, Edwards C, Strang J (1998) The Maudsley Addiction Profile (MAP): a brief instrument for assessing treatment outcome. Addiction 93(12):1857-1867 